\title{
ELEMENTOS ASTRONÓMICOS EN EL MUNDO RELIGIOSO Y FUNERARIO IBÉRICO
}

\author{
ASTRONOMICAL ELEMENTS IN THE RELIGIOUS AND FUNERARY WORLD \\ OF THE ANCIENT IBERIANS
}

CÉSAR ESTEBAN (*)

\begin{abstract}
RESUMEN
Se presenta un análisis arqueoastronómico de la orientación de las estructuras arquitectónicas así como del horizonte que rodea a 16 yacimientos ibéricos de carácter religioso y funerario. Siete de los lugares estudiados (un 44\%) presentan relaciones astronómicas relevantes, principalmente con el orto u ocaso solar en los equinoccios. Por otra parte, se obtiene que la mayoría de los edificios de culto ibéricos no domésticos se encuentran orientados hacia la zona del horizonte donde se producen los ortos del sol (o la luna). Este patrón de orientaciones es similar al mostrado por los templos griegos, los africanos prerromanos y quizás también los púnicos y fenicios, pero diferente al romano y al etrusco.
\end{abstract}

\begin{abstract}
We present an archaeoastronomical study of 16 sacred and funerary Iberian sites. The work is based on the analysis of the orientation of architectural structures as well as the horizon surrounding each site. Seven of the sites surveyed (44\% of them) show relevant astronomical relations, mainly to the sunrise or sunset at the equinoxes. We find that most of the iberian sacred buildings not dedicated to cults of domestic character are oriented to the part of the horizon where the sunrise (or moonrise) takes place. This pattern of orientations is similar to those shown by Greek, preRoman African and perhaps Phoenician and Punic temples, and definetively different to Roman and Etruscan patterns.
\end{abstract}

Palabras clave: Arqueoastronomía. Cultura ibérica. Santuarios. Necrópolis.

(*) Instituto de Astrofísica de Canarias. 38200 La Laguna. Tenerife. Correo electrónico: cel@ll.iac.es

Recibido: 5-III-01; aceptado: 11-II-02.
Key words: Archaeoastronomy. Iberian culture. Sanctuaries . Necropolises.

\section{INTRODUCCIÓN}

El estudio del movimiento y de los cambios de apariencia de los astros permite establecer secuencias temporales de distinta duración como el ciclo día-noche, el de las fases de la Luna (origen del mes), o el ciclo del movimiento del Sol (base del año solar). Está claro, y posiblemente así lo ha sido para el ser humano desde la más remota antigüedad, que el ciclo solar anual regula el ciclo vegetativo de la naturaleza, por lo que la ordenación y previsión de las tareas agrícolas y ganaderas pasa, necesariamente, por la familiarización con los movimientos periódicos del Sol sobre la bóveda celeste. La necesidad de un calendario no obedece sólo a razones fundamentales para la supervivencia de una sociedad, como la ordenación de la actividad económica y productiva, sino también a razones religiosas y rituales y como herramienta de demostración de poder y de "control" de la naturaleza por parte de las jerarquías políticas y religiosas.

Por medio de la arqueología y las fuentes escritas sabemos que la cultura ibérica era una sociedad compleja, dominada por una élite principesca, cuyas actividades económicas principales eran la agricultura y la ganadería. Por otra parte, su génesis se produce debido al contacto directo con culturas orientales como la fenicia y la griega que disponían de calendarios y de un conocimiento astronómico elevado (mucho mejor conocidos en el caso del mundo griego). Por todo ello, no creemos aventurado suponer que la cultura ibérica adquirió unos ciertos conocimientos astronómicos bien por desarrollo propio o por influencia foránea. Es probable 


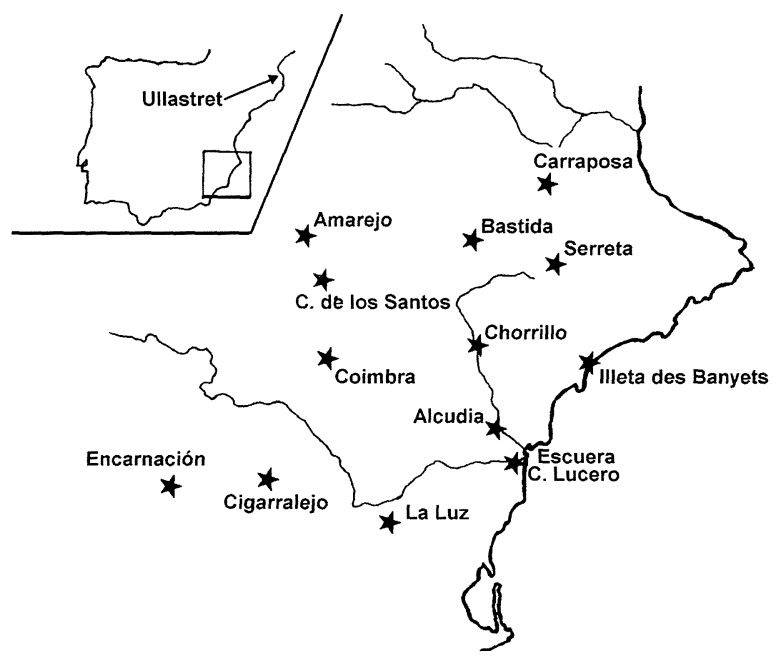

Fig.1. Mapa de localización de los lugares citados en el texto.

que si los aspectos astrales formaron parte del mundo religioso y ritual ibérico, éstos se incluyeran de alguna forma en el diseño de sus construcciones religiosas y funerarias, como de hecho se ha constatado en diferentes culturas de la antigüedad. Esta es la hipótesis que pretendemos comprobar.

El presente artículo es el primer trabajo arqueastronómico extenso realizado hasta la fecha sobre la cultura ibérica. Nos hemos centrado principalmente en el estudio de santuarios y templos (en el futuro incidiremos en las necrópolis) por ser el tipo de yacimientos donde la astronomía podría jugar un papel en su localización y orientación, en razón directa al grado de importancia que tuviesen los aspectos celestes en el rito y en la religión de sus constructores, algo que todavía desconocemos.

\section{METODOLOGÍA Y OBTENCIÓN DE DATOS}

Los datos se obtuvieron directamente sobre los yacimientos y se refieren a dos aspectos: la orientación de los elementos arquitectónicos del yacimiento y la posición relativa de los distintos rasgos llamativos del horizonte local (picos de montañas, muescas, escalones naturales llamativos, otros yacimientos conectados visualmente).

Cada dato de orientación, tanto para un elemento arquitectónico como del horizonte, viene determinado por dos coordenadas: el acimut geográfico (distancia angular horizontal respecto al norte geográfico) y la altura (distancia angular en direc- ción perpendicular a la línea del horizonte). Ambas coordenadas se obtuvieron con una brújula de precisión y un clinómetro portátil. Los acimutes geográficos se obtuvieron a partir de las medidas de la brújula (que proporciona acimutes respecto al norte magnético) corregidas por la declinación magnética $\left(\delta_{\text {mag }}\right)$. En la mayor parte de los casos, la obtención de lă declinación magnética se realizó midiendo el acimut de cotas bien identificadas del horizonte y comparando con el acimut obtenido para esos mismos elementos sobre mapas detallados del Servicio Geográfico del Ejército con escala 1:50.000. En algunos casos particulares, la declinación magnética también se obtuvo mediante la observación de la posición del sol en su orto u ocaso desde el mismo yacimiento, comparando el acimut medido por la brújula y el geográfico esperado según la posición del sol en ese momento (obtenida por interpolación del Almanaque Náutico publicado anualmente por el Real Instituto y Observatorio de la Armada en San Fernando). La declinación magnética es diferente en distintas zonas geográficas, varía con el tiempo y además puede tener variaciones locales dependiendo de los materiales geológicos del suelo, por lo que siempre es aconsejable determinarla en cada yacimiento. Una vez determinada la declinación magnética, el paso de acimut magnético medido, $\mathrm{A}_{2}^{\prime}$, a acimut geográfico, $\mathrm{A}_{\mathrm{z}}$, es simplemente :

$$
A_{z}=A_{z}^{\prime}-\delta_{\text {mag }}
$$

La precisión de las medidas se estima en $\pm 30^{\prime}$ $\left(0,5^{\circ}\right)$ en acimut $\mathrm{y} \pm 10^{\prime}$ en altura $(\mathrm{h})$ para los elementos del horizonte. En el caso de orientación de edificios, la precisión viene determinada principalmente por su estado de conservación y la regularidad de sus paredes y puede llegar a ser de varios grados.

Una vez tenemos los acimutes geográficos de todos los elementos medidos pasamos a su análisis astronómico. El primer paso es relacionar los pares $\mathrm{A}_{\mathrm{z}}$ y h (llamadas coordenadas horizontales) de los elementos escogidos con la posición de ortos u ocasos de astros a través de una sencilla transformación de coordenadas que nos pasa de coordenadas horizontales a coordenadas celestes: ascensión recta $(\alpha)$ y declinación $(\delta)$. La ascensión recta la podemos definir como la "longitud" de un astro respecto a un punto fijo del ecuador celeste, el denominado "punto aries" que corresponde a la intersección (nodo ascendente) entre el plano de la eclíptica con el

T. P., 59, n. ${ }^{\circ} 2,2002$ 


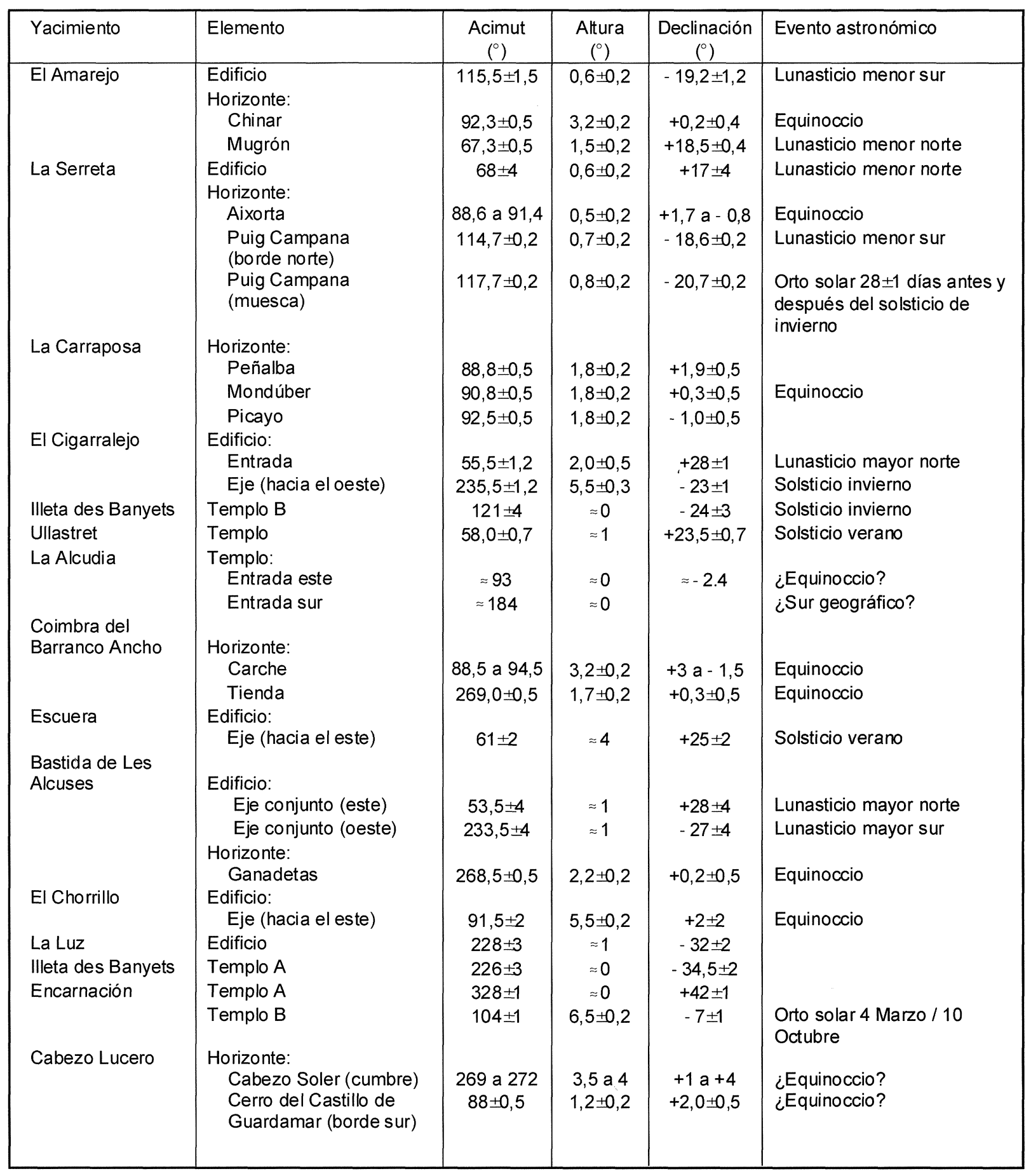

Tab. 1. Datos sobre las orientaciones encontradas.

ecuador celeste. La segunda coordenada, la declinación, es la "latitud" de un punto en el cielo respecto al ecuador celeste. En nuestro análisis sólo nos interesa conocer la declinación, pues esta coordenada es la que nos define trayectorias diferentes sobre la bóveda celeste. En nuestros cálculos hemos considerado los efectos de la refracción atmosférica a alturas pequeñas sobre el horizonte, que afecta a la altura aparente de un astro y a la declinación asociada a éste (Aparicio et al. 2000; Schaefer 2001). 
En nuestro caso, hemos buscado relacionar los elementos del horizonte llamativos o adonde "apuntan" las construcciones con puntos singulares del movimiento periódico del Sol y la Luna, pues son éstos los astros que podrían tener a prio$r i$ una mayor importancia simbólica. En el caso del Sol: los solsticios de invierno (cuando el Sol sale y se pone por su posición más meridional, en el caso de un observador en el hemisferio norte) y de verano (Sol en su posición más septentrional) y los equinoccios (Sol en la línea este-oeste). En el caso de la Luna podemos definir cuatro puntos que corresponden a los lunasticios mayores y menores norte y sur. Son especialmente relevantes los lunasticios mayores, pues corresponden a los puntos más al norte y sur donde podemos ver salir o ponerse a la Luna. No consideramos los planetas, pues al moverse en planos casi coincidentes con el del Sol o la Luna no podemos distinguir de forma clara si una orientación está dirigida realmente hacia un planeta o a uno de los otros dos astros.

En el presente trabajo tampoco consideraremos orientaciones hacia estrellas debido a su ambigüedad. La posición de las estrellas sobre la bóveda celeste cambia con el tiempo debido a la precesión de los equinoccios (que no afecta a las posiciones solares y lunares antes mencionadas) por lo que necesariamente necesitamos la datación del yacimiento y además tenemos que elegir entre multitud de estrellas cuya posición ajusta a la orientación medida en el intervalo de tiempo de uso del edificio. Por otra parte la altura sobre el horizonte a la que comienza o termina a distinguirse una estrella después de su orto (u ocaso) depende fuertemente de su brillo y de las condiciones atmosféricas locales.

El lector no familiarizado con los términos y conceptos astronómicos manejados en esta sección puede encontrar unas sencillas y esclarecedoras introducciones en Aparicio y otros (2000: 19-65), Aveni (1991: 61-153), Belmonte (1999: 265-278) y Krupp (1989: 25-57).

\section{LOS SANTUARIOS IBÉRICOS}

En la presente sección describimos los resultados obtenidos para cada uno de los 15 santuarios ibéricos estudiados. Los yacimientos se han separado en tres grupos. El primer grupo comprende los yacimientos en que hemos encontrado relaciones astronómicas relevantes y donde consideramos una mayor probabilidad de intencionalidad. El segun- do grupo comprende los yacimientos en que hemos encontrado resultados astronómicos que no parecen relevantes, o bien que pudieran serlo bajo ciertas condiciones debido a la falta de datos precisos. A este grupo lo denominamos como de relación astronómica "posible". Finalmente, el tercer grupo engloba a aquellos yacimientos donde no hemos encontrado ninguna relación solar o lunar de interés. Un resumen de los resultados más importantes para cada lugar arqueológico se muestra en la tabla 1.

\subsection{Santuarios con relación astronómica relevante}

\subsubsection{El depósito votivo de El Amarejo}

Este asentamiento ibérico se encuentra situado sobre un cerro amesetado dentro del término municipal de Bonete (Albacete). El yacimiento ha sido excavado parcialmente en varias campañas por Broncano y Blánquez (1985), que encontraron evidencias de que el lugar ya se encontraba ocupado desde la Edad del Bronce. El hallazgo arqueológico más significativo ha sido el de un depósito votivo de unos cuatro metros de profundidad y situado justo en el borde oriental de la cima del cerro (Broncano 1989). El depósito ha suministrado multitud de hallazgos cuya tipología indica claramente su carácter ritual, posiblemente dedicado a una diosa protectora de las actividades femeninas. Inmediatamente al oeste del depósito se encuentran los restos de un edificio de varias estancias que ha sido interpretado por los excavadores como la vivienda del personal encargado del santuario, aunque AlmagroGorbea y Moneo (2000: 51) comentan que el denominado departamento 1 del complejo pudiera corresponder incluso a la cella del santuario. El análisis de la cerámica encontrada en el yacimiento indica que el depósito se utilizó hasta finales del siglo III o principios del II a.C. (Broncano 1989: 34). Resultados arqueoastronómicos preliminares han sido publicados en Esteban $(1999,2001)$.

El horizonte visible desde el emplazamiento del depósito votivo es muy amplio, abarcando toda la mitad oriental. Sin lugar a dudas el elemento topográfico más llamativo que se divisa es la cercana montaña Chinar, situada a $2,7 \mathrm{~km}$ de distancia y con unos $200 \mathrm{~m}$ de altura sobre la llanura circundante (ver Lám. I). El análisis de las coordenadas de la cumbre de la montaña nos indicó que la salida del sol en o muy cerca de los equinoccios debería pro-

T. P., 59, n. ${ }^{\circ} 2,2002$ 


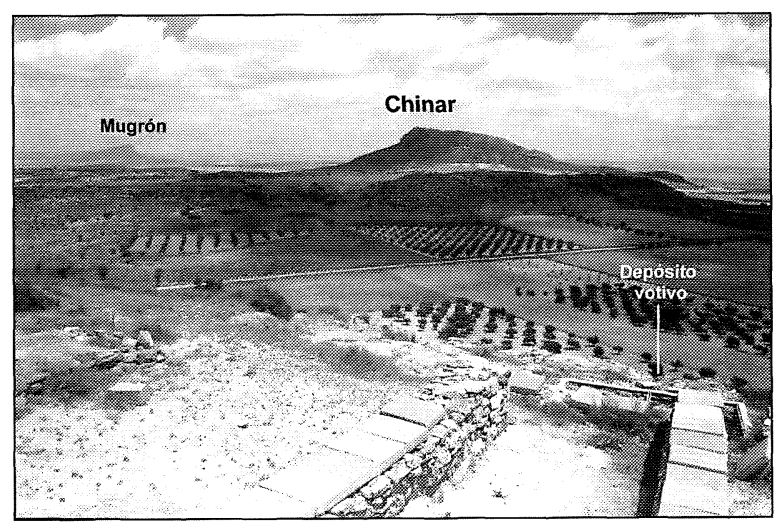

Lám. I. Vista parcial del horizonte oriental desde el depósito votivo de El Amarejo. La montaña Chinar es el elemento más llamativo.

ducirse justo sobre este elemento. Visitamos el lugar al amanecer durante varios días alrededor del equinoccio de primavera de $2000(19,20$ y 21 de marzo). Debido al mal tiempo sólo fue posible observar la salida del Sol el día 19 de marzo. Dicha fecha correspondía con el día previo al equinoccio de primavera, por lo que el Sol, al amanecer, se encontraba ligeramente al sur $\left(\delta=-0^{\circ} 25^{\prime}\right.$, algo menos de un diámetro solar) de su posición en el equinoccio $\left(\delta=0^{\circ}\right)$. En este momento pudimos contemplar y fotografiar el disco solar emergiendo ligeramente al sur de la cúspide de Chinar (ver Lám. II). Estimamos que al amanecer del 20 de marzo (día del equinoccio) el Sol saldría aproximadamente un diámetro solar más al norte, por lo que coincidiría con la cumbre de la montaña. Otro fenómeno más llamativo si cabe se produciría al amanecer de los días 21 y 22 de marzo (los dos días inmediatamente posteriores al equinoccio de primavera). En estos momentos, el Sol se habría desplazado cerca de dos y tres diámetros solares respectivamente más al norte de su posición observada del 19 de marzo $(\delta=$ $+0^{\circ} 22^{\prime}$ y $+0^{\circ} 46^{\prime}$ para cada fecha), por lo que el orto se produciría cercano a la parte inferior de la ladera norte de Chinar y el disco solar iría "deslizándose" semiescondido de forma prácticamente tangente a dicha ladera (la trayectoria del sol sigue un ángulo de unos $39^{\circ}$ respecto de una línea vertical imaginaria, mientras que la inclinación de la ladera es de unos $48^{\circ}$, ver Lám. II). Hay que tener en cuenta que el sol pasa dos veces al año por estos puntos del horizonte, por lo que en el equinoccio de otoño (alrededor del 22 de septiembre) tendríamos los mismos fenómenos aunque el desplazamiento de la posición del orto solar sobre el horizonte de un

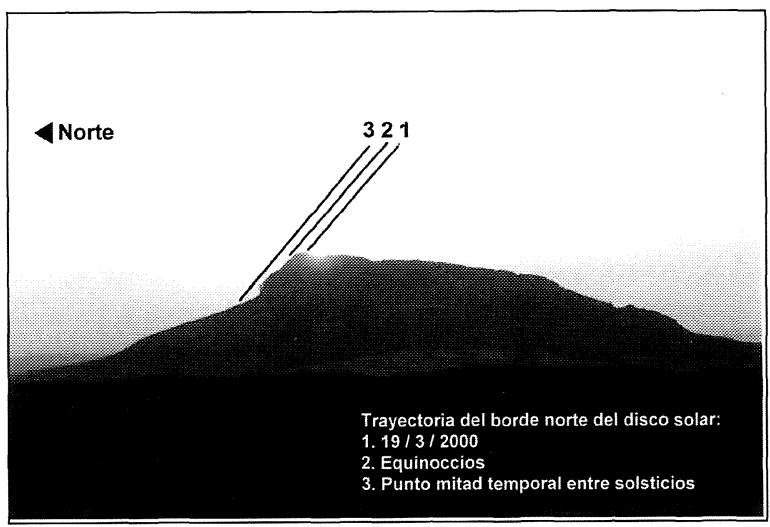

Lám. II. Representación de la trayectoria del borde septentrional del disco solar en tres días consecutivos alrededor del equinoccio, tal y como puede verse desde el depósito votivo de El Amarejo. El 19 de marzo (fecha en que se tomó la fotografía), día anterior al equinoccio de primavera, el sol sale ligeramenté (algo menos de un diámetro solar) al sur de la cúspide de la montaña. El 20 de marzo, equinoccio de primavera, el sol sale justo por el borde norte de la montaña. El 21 de marzo, día posterior al equinoccio y punto medio temporal entre ambos solsticios (ver texto), el sol sale realizando un llamativo «deslizamiento» sobre la ladera norte de Chinar; la trayectoria es prácticamente tangente a la ladera.

día para otro se realiza en sentido contrario: de norte a sur. En este segundo momento del año tendríamos la siguiente secuencia: los dos días inmediatamente anteriores al equinoccio el Sol saldría por la ladera norte realizando el llamativo "deslizamiento" sobre ella; el día del equinoccio el Sol aparecería por la cumbre y, finalmente, el día posterior, el amanecer se produciría de forma similar a la observada el 19 de marzo. Hay que hacer notar que estos fenómenos no serían visibles de igual forma y en las mismas fechas si uno se mueve varias decenas de metros al norte o al sur de la posición del depósito votivo, por lo que, dentro de la hipótesis astronómica, la observación del fenómeno pudo ser el motivo (o uno de los motivos) de la elección del emplazamiento del santuario. Hay que hacer notar que la posición de los equinoccios no ha variado desde la época ibérica hasta la actual, por lo que el fenómeno sigue siendo visible de forma prácticamente idéntica.

Los hechos relatados nos sugieren que quizás el santuario de El Amarejo tuvo una utilidad astronómica para la determinación de los equinoccios o que en él se aprovechaba la hierofanía astronómica como un elemento del ritual que se llevaba a cabo en ese momento del año. Afortunadamente, los resultados de las excavaciones nos dan algunos argu- 
mentos que apoyan nuestra hipótesis. En primer lugar, los excavadores encontraron que las ofrendas incineradas del depósito estaban distribuidas en capas aparentemente periódicas, lo que sugiere que los rituales se realizaban en momentos determinados del año (Broncano 1989: 33). Uno de los objetos más comunes en las ofrendas eran las bellotas que, según Broncano (1989: 33), conservaban todavía su "caperuzón", lo que indica que estaban aún algo verdes o a medio madurar. Teniendo en cuenta el desarrollo de las bellotas en el área que nos ocupa, dicho autor sugiere que el ritual del santuario se llevaría a cabo anualmente en otoño, posiblemente a principios de octubre. Este resultado es consistente con nuestra propuesta en el caso de que el ritual se realizase en el equinoccio de otoño o una fecha muy cercana a éste.

Finalmente, queremos comentar que AlmagroGorbea y Moneo (2000: 49) indican que el edificio anexo al depósito votivo está orientado hacia el orto solar en el solsticio de invierno. Este resultado se basa en el azimut magnético, lo que proporciona una gran incertidumbre. Nuestras medidas corregidas por declinación magnética indican que las paredes del edificio anexo al depósito apuntan, dentro de la incertidumbre, a la posición de la luna en el lunasticio menor sur, aunque esta relación puede ser casual dado lo difícil que resulta determinar esta posición lunar y su escaso interés práctico (Aparicio et al. 2000: 32-36; Belmonte 1999: 268270).

\subsubsection{El santuario de La Serreta}

Esteban y Cortell (1997) encontraron un posible marcador de los equinoccios en este yacimiento, pues el orto solar se produce sobre la Sierra de Aixorta (elemento distante del horizonte oriental) durante unos 6 días alrededor de los equinoccios. Las imágenes obtenidas indican que el orto se realiza sobre el centro del perfil visible de la citada sierra cuando el Sol se encuentra a $\delta \approx+0^{\circ} 45^{\prime}$, posición que alcanza unas 45 horas después del momento exacto del equinoccio de la primavera o después del equinoccio de otoño (ver Lám. III), momento en el que también se produce el "deslizamiento" del disco helíaco sobre la ladera norte de la montaña Chinar para un observador situado en El Amarejo.

En distintos trabajos recientes (Gusi 1997; Olcina Doménech et al. 1998) se plantearon dudas de que el basamento atribuído al edificio sacro por

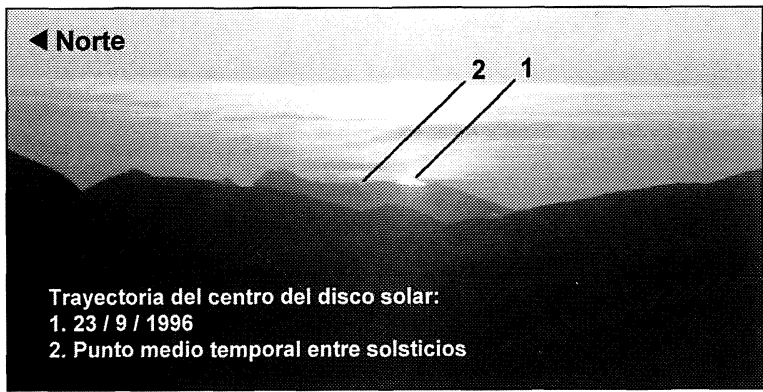

Lám. III. Trayectoria del centro del disco solar el día del equinoccio de otoño de 1996 (23 de Septiembre) y la correspondiente al punto medio temporal entre ambos solsticios. En este último caso, el orto solar se produce justo en el centro de la zona visible de la Sierra de Aixorta (foto: Emilio Cortell).

Llobregat y otros (1992) correspondiera realmente al santuario, proponiéndose que éste se encontraba en las cercanías del vértice geodésico situado en la cumbre de la montaña (situado a unos $70 \mathrm{~m}$ al oeste). El estudio de Esteban y Cortell (1997) asumía la propuesta de Llobregat y otros. En este punto es necesario comentar que los resultados sobre el marcador equinoccial sobre la Sierra de Aixorta, así como los demás resultados referidos a ortos solares o lunares sobre elementos del horizonte, son aplicables también desde cualquier punto de observación situado sobre la cresta de la montaña de La Serreta debido a su peculiar orientación casi paralela al eje este-oeste y a la lejanía del horizonte, tal y como como pudimos comprobar con medidas directas.

\subsubsection{El santuario de La Carraposa}

Este santuario ibérico al aire libre se encuentra en la ladera sureste del cerro de La Carraposa. El horizonte levante se encuentra completamente libre y domina visualmente toda la hoya de Xàtiva, incluída la antigua Saitabi. Las prospecciones y excavaciones arqueológicas llevadas a cabo desde 1997 a 1999 por Pérez Ballester y Borredá Mejías han proporcionado más de un centenar de fragmentos de terracotas representando en su mayor parte a équidos. Los materiales indican que la cronología del yacimiento es bastante tardía, entre el siglo II y primera mitad del I a.C. (Pérez Ballester y Borreda Mejías, e.p.).

El análisis completo de este yacimiento se presenta en Pérez Ballester y Borreda Mejías (e.p.). El

T. P., 59, n. ${ }^{\circ} 2,2002$ 
resultado más llamativo es que el orto solar cerca de los equinoccios $\left(\delta \approx+0^{\circ} 35^{\prime}\right)$ se produce justo sobre el pico del Mondúber, la montaña más alta del horizonte oriental. Además, este pico se encuentra flanqueado por otros dos también cónicos, más cercanos y de altura angular similar: El Pico de Peñalba, al norte, y el Alto del Picayo, al sur; ambos picos se encuentran separados casi la misma distancia angular (alrededor de $2^{\circ}$ y $1,7^{\circ}$ respectivamente) del Mondúber, lo que hace que 4 días antes y después de cada uno de los equinoccios el sol tenga su orto por alguno de estos picos adyacentes, por lo que tendríamos unos excelentes indicadores de la proximidad de los equinoccios. Este marcador equinoccial es muy similar al encontrado en La Serreta, que se encuentra a unos escasos $36 \mathrm{~km}$ de distancia en línea recta.

\subsubsection{El santuario de El Cigarralejo}

El santuario de El Cigarralejo está situado sobre un cerro cercano a la importante necrópolis del mismo nombre dentro del término municipal de Mula (Murcia). El edificio, excavado por Cuadrado Díaz (1950), tiene planta rectangular alargada y consiste en varias estancias conectadas por un pasillo común. Su entrada, de pequeñas dimensiones, se encuentra en el extremo noreste del edificio. Durante las excavaciones se encontró un depósito subterráneo en el extremo suroeste del santuario que proporcionó numerosas esculturas de caballos y otros equinos, así como algunas figuras humanas. El santuario se ha datado entre los siglos IV y II a.C.

La orientación del eje mayor del edificio muestra una doble relación astronómica (ver Lám. IV). La dirección hacia la que "mira" la entrada del edificio sobre el horizonte local corresponde al orto de un cuerpo celeste con $\delta=+28 \pm 1^{\circ}$ coincidente, dentro de la incertidumbre, con la posición de la Luna en el lunasticio mayor norte, el punto más septentrional que puede alcanzar el orto de la Luna $\left(\delta=+28,8^{\circ}\right.$ en el siglo III a.C.). Por otra parte, en la dirección opuesta (suroeste) la altura del horizonte es mayor, pues el eje mayor del edificio apunta hacia la cumbre del cerro, por lo que el santuario se encuentra alineado también al ocaso de un cuerpo celeste con $\delta=-23 \pm 1^{\circ}$, que coincide con la posición del sol en el solsticio de invierno $\left(\delta=-23,7^{\circ}\right)$. Por razones de geometría orbital, la Luna llena más cercana al lunasticio mayor norte se alcanza también

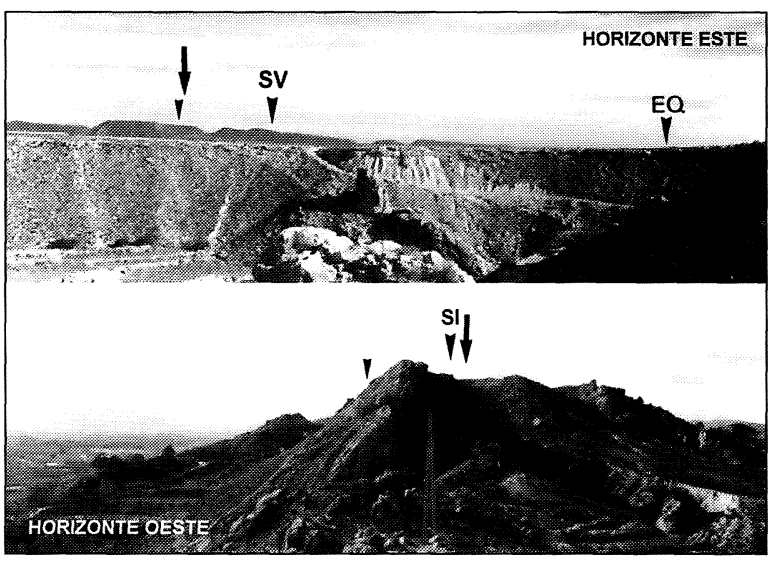

Lám. IV. Vista parcial de los horizontes oriental y occidental que rodean al santuario extraurbano de El Cigarralejo. Las flechas rotuladas como EQ, SV y SI indican la posición del orto u ocaso solares en los equinoccios, el solsticio de verano y el solsticio de invierno respectivamente. Las flechas largas indican las posiciones adonde apunta el eje mayor del edificio en la dirección de ambos horizontes. Las flechas cortas indican el lugar donde se produce el orto lunar en el lunasticio mayor norte (horizonte este) y el ocaso lunar en el lunasticio mayor sur (horizonte oeste).

en las cercanías del solsticio de invierno, por lo que, en dicho momento, tendríamos ambos cuerpos celestes alineados simultáneamente con el eje mayor del edificio. Este doble alineamiento se alcanzaría cada 18,6 años (ciclo de regresión de la línea de los nodos Aparicio et al. 2000: 32).

\subsubsection{Templo B de la Illeta des Banyets}

El poblado ibérico de la Illeta des Banyets se encuentra situado en una pequeña península en $\mathrm{El}$ Campello (Alicante). Dentro del yacimiento se encontraron dos posibles templos que han sido excavados por Llobregat (1985). El edificio de mayor tamaño y complejidad, el denominado templo A, será discutido más adelante (sección 3.3.2). El más pequeño, el templo $\mathrm{B}$, situado a escasos metros al oeste del anterior, es una estructura a cielo abierto con planta cuadrada y con su entrada orientada hacia el horizonte sureste. Presenta claros paralelos semíticos (Llobregat 1985; Almagro-Gorbea 1996: 95-96). Según Llobregat, el templo estuvo dedicado a una diosa de los muertos y la fecundidad. $\mathrm{La}$ cronología del conjunto iría desde el último cuarto del siglo $\mathrm{V}$ hasta finales del siglo IV a.C.

Nuestras medidas nos indican que la entrada del templo B apunta al orto de un cuerpo celeste con 
$\mathrm{d}=-24 \pm 3^{\circ}$, intervalo que engloba la posición del Sol en el solsticio de invierno $\left(\delta=-23,7^{\circ}\right.$ en el siglo IV a.C.). Creemos interesante comentar que el templo púnico de la ciudad costera de Kerkouane (datado entre los siglos V y II a.C.), y que podría ser un buen paralelo formal al templo alicantino (AlmagroGorbea y Moneo 2000: 138), muestra una orientación bastante similar $\left(\delta=-29 \pm 3^{\circ}\right.$, Esteban et al. 2001).

\subsubsection{El templo de Ullastret}

Este edificio de culto se encuentra en la parte más alta del poblado ibérico de Ullastret situado en el Puig de Sant Andreu (Gerona). Es el único yacimiento ibérico fuera del área sureste del que tenemos medidas directas. Se trata de un templo rectangular in antis, con su entrada orientada al este y que fue destruido en el siglo III a.C. (Miró i Alaix 1990). En su interior se encontraron máscaras votivas relacionables con motivos y costumbres griegas. Miró i Alaix (1990) sugiere que el templo estaría dedicado a alguna divinidad relacionable con cultos a la fertilidad.

Según nuestras medidas, la disposición y orientación de las distintas paredes del templo se realizó de forma muy cuidadosa. La entrada del edificio apunta al orto de un astro situado a $\delta=+23,5 \pm 0,7^{\circ}$, posición que coincide, dentro de las incertidumbres, con la posición del Sol en el solsticio de verano en la época de uso del templo $\left(\delta=+23,7^{\circ}\right)$.

\subsection{Santuarios con relación astronómica posible}

\subsubsection{El templo de La Alcudia}

En el actual yacimiento de La Alcudia de Elche tenemos uno de los templos ibéricos conocidos más antiguos del sureste, de clara tipología semítica (Ramos Fernández 1995: 13). El edificio se encontró debajo de las ruinas de una basílica paleocristiana, en el extremo sur del yacimiento. Según Ramos Fernández (1995), el santuario estaría dedicado al culto de una divinidad femenina de la fecundidad. En la lámina $\mathrm{V}$ mostramos una fotografía de la reconstrucción del templo que se levanta apenas unos metros al sur de su verdadero emplazamiento. El edificio presenta dos entradas, una orientada al sur, aparentemente la principal, y otra abierta en una estancia o torre adosada al extremo noreste del templo y que mira hacia el este. Debajo de este segundo acceso se encontró un depósito fundacional (favissa) con diverso material cerámico ibérico arcaico, lo que le confiere una especial relevancia, quizás ritual. La torre o habitación adosada dispone de otra abertura que conecta con la estancia principal del templo (fase B, reciente) o a una tercera estancia intermedia en la fase A (arcaica). Todas estas aberturas (dos en la fase B y tres en la fase A) se encuentran orientadas hacia levante.

Debido a que el edificio original se volvió a enterrar, fue imposible obtener medidas de la orientación del propio edificio. En su lugar disponemos de varios datos indirectos: la reconstrucción (de la que no podemos estar seguros si se respetó estrictamente la misma orientación que el original), los planos publicados (en donde no se indica si el norte es el magnético o el verdadero) y la indicación verbal por parte del excavador de que la orientación del templo es coindidente con la de la basílica paleocristiana que se construyó encima (Ramos Fernández 1995, comunicación personal). Tomando como referencia esta última indicación, asignamos el promedio de la orientación de las paredes norte y sur de la basílica (eje mayor del conjunto) como orientación de las paredes norte y sur del templo ibérico. Como resultado obtenemos que la entrada abierta en el anexo del templo está orientada $\mathrm{a}_{\mathrm{z}}=96,5^{\circ}$. Aunque la dirección a la que apunta no es estrictamente equinoccial, no podemos eliminar la posibilidad de alguna relación con este evento astronómico (ver Lam. V). Por ejemplo, el desplazamiento anual de la proyección de la luz solar sobre elementos de la pared oeste (donde pudo existir un banco corrido con esculturas según Ramos Fernández) pudo haber sido utilizado para marcar fechas importantes del calendario.

\subsubsection{El santuario de Coimbra del Barranco Ancho}

La existencia de un santuario en el yacimiento ibérico de Coimbra del Barranco Ancho (Jumilla, Murcia) fue propuesta por García Cano y otros (1991-92) debido al hallazgo de fragmentos de figuras de terracota repartidas por las laderas de un cabezo al este del Cerro del Maestre, donde se sitúan un poblado y tres necrópolis. Dichas figuras parecen corresponder a versiones locales de los pebeteros con representación de cabeza de Deméter tan exten-

T. P., 59, n. ${ }^{\circ} 2,2002$ 


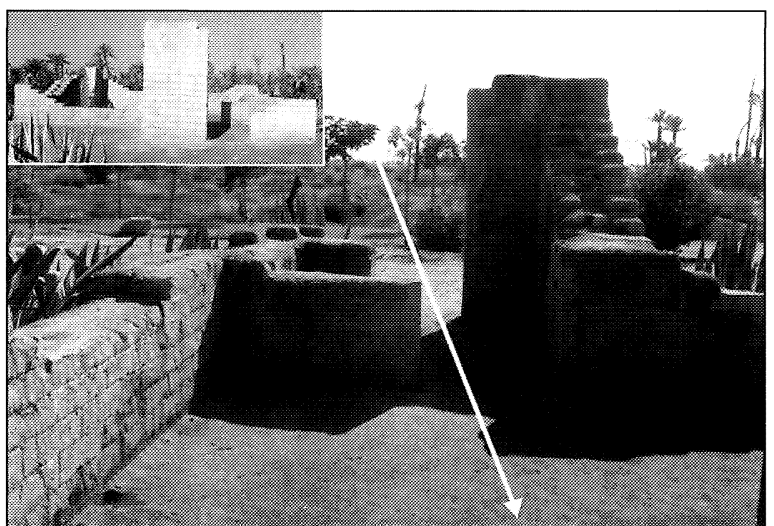

Lám. V. Vista parcial de la reconstrucción del templo ibérico de La Alcudia de Elche. La fotografía muestra la entrada oriental vista desde la pared interior oeste, sobre la que existía un banco corrido. En la esquina inferior derecha se ve parte del altar. Esta entrada está orientada cerca del orto solar de los equinoccios. La flecha muestra que el sol del amanecer pudo iluminar el interior del templo a través de la abertura en fechas cercanas a los equinoccios. El seguimiento anual de la posición de la zona iluminada sobre la pared oeste pudo haber sido utilizado como indicador calendárico. El cuadro superior muestra la fachada este del templo.

didos por todo el área ibérica. La cronología que se asigna a las figuras va desde mediados del siglo IV a principios del siglo II a.C. Según las indicaciones de García Cano y otros (1991-92) lo más probable es que los restos provinieran de "una favissa o acaso vertedero" situado en el extremo sur del cabezo.

Debido a la inexistencia de restos arquitectónicos sólo pudimos realizar el análisis de los elementos relevantes del horizonte. En primer lugar obtuvimos medidas desde el promontorio donde se encontraron las terracotas, aunque no encontramos ningún resultado de interés. Nos llamó la atención que a unos $200 \mathrm{~m}$ al norte del promontorio y sobre el punto más alto del mismo cabezo se encuentra una cruz. Teniendo en cuenta que en algunos casos estos elementos son testigos de antiguos actos de cristianización de lugares de culto antiguos, también realizamos medidas sobre dicho punto. El horizonte que se divisa desde la cruz es más amplio, sobre todo hacia el este, en donde se encuentra el Carche, la montaña más alta de toda la zona $(1.372$ m s.n.m.), y que no es visible desde el promontorio del sur. Las medidas tomadas indican que la zona superior y relativamente plana del perfil de El Carche ocupa una franja de declinación que va desde $+3^{\circ}$ a $-1,5^{\circ}$, correspondiendo este último valor a la cumbre de la montaña (ver Lam. VI). Es significa-

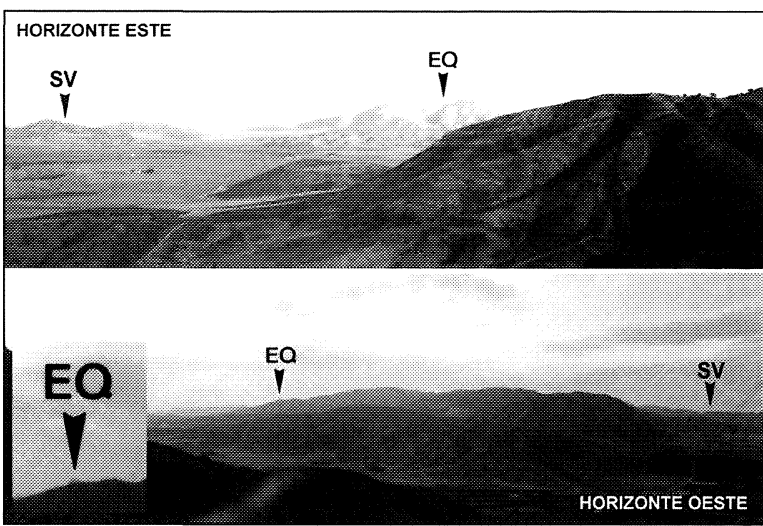

Lám. VI. Vista parcial de los horizontes oriental y occidental que rodean el lugar de localización que proponemos para el santuario al aire libre de Coimbra del Barranco Ancho (ver texto). Las flechas rotuladas como EQ y SV indican la posición del orto u ocaso solares en los equinoccios y el solsticio de verano respectivamente. El orto solar en los equinoccios y fechas cercanas a éste se produce sobre la montaña de El Carche, la más alta de toda la zona. El ocaso en los equinoccios $\left(\mathrm{d} \approx+0,3^{\circ}\right)$ se produce sobre el lejano Pico de Tienda que se encuentra en segundo término (ver ampliación en el recuadro de la izquierda).

tivo que el punto medio de estos dos valores corresponde a $+0,75^{\circ}\left(+0^{\circ} 45^{\prime}\right)$ justo la posición que indican también los marcadores de La Serreta y La Carraposa. Por otra parte, hacia el oeste podemos ver el pequeño Pico de Tienda sobresaliendo ligeramente por detrás del perfil suave y extenso de la Sierra del Molar, nuestras medidas indican que sobre este pico (que apenas ocupa $1 / 3^{\circ}$ ) se produce el ocaso de un astro con $\delta=+0,3^{\circ}$, por lo que el ocaso solar en los equinoccios también se produciría por un lugar relativamente llamativo del horizonte proporcionando un marcador adicional muy preciso.

Como vemos, el lugar tiene un potencial astronómico significativo, siempre y cuando la localización exacta del santuario fuese cercana a la cumbre del cabezo donde actualmente se encuentra la cruz y no donde se encontraron las terracotas. Este hecho es obviamente un problema, pero la presencia de vertederos fuera de los edificios de culto es algo común en el mundo ibérico, como por ejemplo en La Serreta o en Castellar de Santisteban.

\subsubsection{El posible edificio cultual de La Escuera}

Este edificio, situado en el poblado ibérico de La Escuera (San Fulgencio, Alicante), fue excavado 
parcialmente por Nordström (1967). La cronología asignada al poblado va desde los siglos IV al II a.C. (Abad y Sala 1997). La estructura del presunto santuario es muy complicada con no menos de 8 departamentos y cámaras semisubterráneas.

Aunque Abad y Sala (1997) comentan la carencia de un eje lineal característico en el edificio, creemos que los distintos departamentos se encuentran orientados siguiendo un entramado regular según dos ejes perpendiculares relativamente bien definidos. Medimos la orientación de aquellas paredes mejor conservadas y regulares, que coincidieron con las paredes norte, este y oeste del "departamento e" y el lienzo de muro perpendicular que sale de su pared oriental y se introduce en el denominado "departamento f". No conocemos donde se encontraba la entrada del recinto pero si, como hipótesis, admitimos que se encontraba situada a levante, como ocurre en la mayor parte de los santuarios ibéricos (ver sección 4), encontramos que el eje suroeste-noreste apuntaría al orto de un cuerpo celeste con $\delta=+25$ $\pm 2^{\circ}$, orientación consistente con el orto solar en el solsticio de verano $\left(\delta=+23,7^{\circ}\right)$. Hay que hacer notar que esta misma orientación es la que presenta el templo de Ullastret y similar a la que muestran el santuario de El Cigarralejo y el conjunto 5 de La Bastida.

\subsubsection{El santuario del Cerro de los Santos}

El santuario del Cerro de los Santos (Montealegre del Castillo, Albacete), conocido desde mediados del siglo XIX, es uno de los ejemplos más representativos de lugares de culto ibéricos, aunque nada nos queda del edificio original. En nuestras repetidas visitas al lugar no hemos encontrado ningún resultado de interés astronómico relacionado con el horizonte que rodea el yacimiento. El plano del antiguo templo publicado por Savirón (1875) y reproducido en múltiples trabajos posteriores muestra que el eje mayor del templo estaba orientado prácticamente en dirección oeste-este, con la entrada apuntando a levante, aunque desconocemos la precisión con el que se realizó el plano y si el norte que se representa es el geográfico o el magnético.

\subsubsection{Posible edificio cultual de La Bastida de Les Alcuses}

El denominado conjunto 5 del poblado ibérico de La Bastida de Les Alcuses (Moixent, Valencia) ha sido interpretado como posible edificio cultual por Díes Cusi y Álvarez García (1997). Se halla situado en la parte más alta del yacimiento y se divide en dos áreas, una sin edificar, al sur y delimitada por un muro perimetral, y otra al norte donde se encuentran varios departamentos adosados, algunos de ellos con entradas orientadas al sureste. Los excavadores distinguen dos fases de construcción, ambas situadas en el siglo IV a.C. Una característica del complejo que resaltan especialmente Díes y Álvarez es la ausencia de construcciones en extensas áreas adyacentes hacia el este y el oeste del edificio, lo que hace del edificio un punto apropiado de observación.

Los departamentos que mejor se conservan del conjunto (y posiblemente los principales: número 63,64 y 65 ) presentan su entrada hacia el sur-sureste, demasiado al sur para apuntar hacia el orto del Sol o de la Luna, sin embargo la pared norte del conjunto y la perpendicular hacia el este de las paredes de los tres departamentos muestran una orientación hacia el orto de un astro con $\delta=+28 \pm 4^{\circ}$, la misma orientación que el edificio del santuario de El Cigarralejo y englobando el punto del orto lunar en el lunasticio mayor norte. Es de hacer notar que, según la hipótesis de reconstrucción de Díes Cusi y Âlvarez García (1997), el complejo tendría dos entradas en el recinto perimetral murado, una mirando hacia el este y otra al oeste, por lo que el acceso exterior oriental podría apuntar hacia el evento astronómico citado. Por otra parte, el acceso situado a poniente, por simetría, estaría apuntando al ocaso de un astro con $\delta=-27 \pm 4^{\circ}$, coincidente con el lunasticio mayor sur de la Luna.

El análisis del horizonte fue parcial debido a que el poblado está cubierto por un bosque de pinos, lo que dificulta la observación de buena parte de éste. De cualquier forma el horizonte este no parece presentar elementos topográficos relevantes según los planos manejados. Hacia el oeste pudimos observar una franja de horizonte donde encontramos que la cumbre del Cerro Las Ganadetas, de $1.004 \mathrm{~m}$ de altura s.n.m., aunque no demasiado llamativo, coincide prácticamente con el ocaso solar en los equinoccios $\left(\delta=+0,2^{\circ}\right)$.

\subsubsection{Edificio singular de El Chorrillo}

Este yacimiento se encuentra situado en la confluencia de los términos municipales de Sax, Petrer y Elda (Alicante). Se trata en un edificio aislado de

T. P., 59, n. $^{\circ} 2,2002$ 
planta rectangular que ocupa la zona meridional y más alta de un pequeño cerro situado a escasos metros del río Vinalopó. Márquez Villora y otros (1999) encuentran que el lugar ya fue utilizado desde la segunda mitad del siglo VI a.C. y a lo largo del $\mathrm{V}$ a.C., aunque la mayoría de los restos corresponden al siglo IV a.C.

El horizonte que se divisa desde el lugar es muy amplio y muy rico en elementos llamativos. De cualquier forma, sólo hemos encontrado que el orto del solsticio de invierno se produce en una depresión del horizonte formada por la intersección de dos montañas. La orientación de los restos del edificio, básicamente dos paredes perpendiculares, parece realizada con bastante cuidado. La orientación general sigue los ejes geográficos N-S E-O con una desviación de $1,5^{\circ}$, del orden de la incertidumbre $\left( \pm 2^{\circ}\right)$. Este dato nos indica que la orientación del edificio es similar al de La Alcudia y la distribución general de las tumbas de la necrópolis de Cabezo Lucero, resultado que refuerza el posible uso ritual del edificio. Más detalles sobre los resultados obtenidos para este yacimiento pueden encontrarse en Esteban y Poveda (e.p.).

\subsection{Santuarios sin relación astronómica aparente}

\subsubsection{El santuario de La Luz}

El yacimiento ibérico de La Luz (Murcia) se encuentra sobre un cerro que ha proporcionado un complejo de estructuras, exvotos de bronce y un templo en la cumbre cristianizado con una cruz. A raíz de las excavaciones, Lillo Carpio (1993-1994) indica que el templo fue construido en el tránsito de los siglos III a II a.C. y posiblemente sobre alguna estructura ibérica de culto anterior. Según el excavador, la disposición de los accesos y del contexto inducen a pensar que la entrada del templo estaba orientada a poniente. El análisis del horizonte no proporcionó ninguna relación solar o lunar evidente. La dirección hacia donde apunta la supuesta entrada del templo corresponde con el ocaso de un astro con $\delta=-32 \pm 2^{\circ}$, demasiado al sur para estar relacionado con el Sol y la Luna.

\subsubsection{El "templo" A de Illeta des Banyets}

La interpretación de este edificio es problemática, mientras su excavador lo clasifica como edi- ficio de culto (Llobregat 1985), Almagro Gorbea y Domínguez (1988-1989) lo interpretan como regia. La entrada del edificio se encuentra apuntando al ocaso de un astro con $\delta=-34,5 \pm 2^{\circ}$, similar a la orientación del templo de La Luz, sin relación posible con el Sol o la Luna.

\subsubsection{El santuario de La Encarnación}

El santuario ibérico de la Encarnación se encuentra situado en el Cerro de La Ermita (Caravaca, Murcia). Ha proporcionado diversas piezas escultóricas realizadas en arenisca (Ruano y San Nicolás del Toro 1990) cuya cronología es amplia, desde el siglo IV al I a.C. No se conocen restos constructivos ibéricos, pero sí las ruinas de dos templos de época romana tardo-republicana (Ramallo 1991). La orientación de estos dos templos es muy diferente, el mayor de ellos, el denominado templo A (que se encuentra debajo de una ermita construida en el siglo XVI), apunta al noroeste, hacia el ocaso de un astro con $\delta=+42 \pm 1^{\circ}$, sin relación solar o lunar posible. Por otra parte, el templo $B$ se encuentra orientado hacia el sureste, hacia el orto de un astro con $\delta=-7 \pm 1^{\circ}$, esta posición es alcanzada por el sol en fechas poco significativas astronómicamente, alrededor del 4 de marzo y del 10 octubre (en el año de referencia del 300 a.C. y según el Calendario Gregoriano actual). Lo curioso es que esta dirección coincide con el único rasgo llamativo del horizonte oriental, la cumbre del Pico Cabras, que aparece en segundo término ocupando unos $12^{\circ}$ sobre el perfil cercano del Cerro de La Ermita.

\section{EL PATRÓN DE ORIENTACIÓN DE LOS EDIFICIOS DE CULTO IBÉRICOS}

En la figura 2a representamos datos sobre la orientación de las entradas de una fracción importante de los edificios de culto ibéricos conocidos. Incluimos tanto orientaciones obtenidas en el presente trabajo como otras recopiladas a partir de planos publicados en la literatura (Alorda Park, santuario A: Belarte y Sanmartí 1997: fig. 7; Azaila, templo A: Almagro Gorbea y Moneo 2000: 78; Burriac: Barberà y Pascual 1979-80: fig. 1; Cancho Roano: Celestino Pérez et al. 1996: 32; Castellet de Bernabé, departamento 2: Moret 1996: fig. 71; Cerro de los Santos: Savirón 1875; El Oral, habitación 

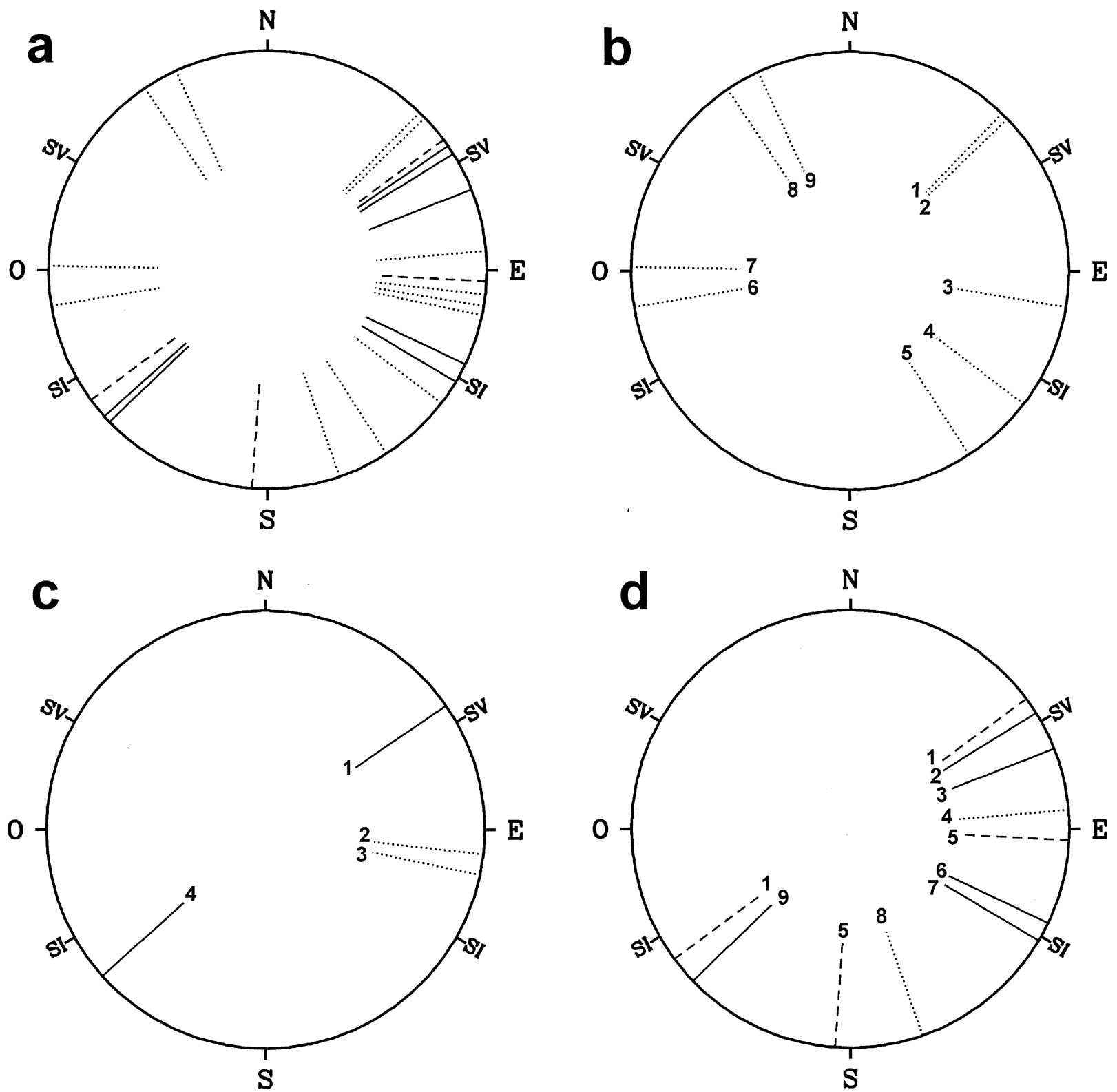

Fig. 2. Diagramas de orientación de distintos conjuntos de edificios de culto ibéricos. El diagrama 2a muestra todos los estudiados en este trabajo (líneas continuas y discontinuas) y otros recopilados de planos e información publicados (líneas de puntos). Las líneas discontinuas representan la orientación de edificios que presentan dos entradas, por lo que cada santuario de este tipo viene definido por dos líneas. La identificación de cada uno de ellos se recoge en el resto de diagramas de esta figura. El diagrama $2 b$ muestra la orientación de edificios de culto clasificados como «domésticos gentilicios» y «dinásticos gentilicios» por Almagro-Gorbea y Moneo (2000), insertos en el tejido urbano de los poblados (1-Moleta del Remei; 2-El Oral; 3-Llíria; 4- Molí d'Espígol; 5-Puntal dels Llops, estancia 1; 6-Burriac; 7- Castellet de Bernabé; 8- Puntal dels Llops, estancia 14; 9-Alorda Park). El diagrama 2c recoge la orientación de santuarios extraurbanos (1-El Cigarralejo; 2-Cerro de los Santos; 3-Cancho Roano; 4-La Luz). Finalmente, el diagrama 2d muestra la orientación de los santuarios urbanos no clasificados como «gentilicios» por Almagro-Gorbea y Moneo (2000), esta muestra incluye los «recintos sacros», de tipo «clásico», «dinástico clientelar» y «de entrada» (1-Bastida de Les Alcuses; 2-Ullastret; 3-La Serreta; 4Azaila; 5-La Alcudia; 6-El Amarejo; 7-Illeta des Banyets, templo B; 8-Torreparedones; 9-Illeta des Banyets, templo A). 
IIIJ: Abad y Sala 1997: fig. 1; Moleta del Remei, recinto 7: Belarte y Sanmartí 1997: fig. 2, 146; Molí d'Espígol, estancia 65: Maluquer 1986: 27; Puntal dels Llops, estancias 1 y 14: Bonet et al. 1986: 323; Sant Miquel de Llíria, estancia 14: Bonet 1995: fig. 28; Torreparedones: Fernández Castro y Cunliffe 1988: fig. 1). En los casos donde se ha obtenido la orientación a partir de planos la incertidumbre puede ser importante, pues en ellos no se suele indicar si la orientación se refiere al norte geográfico o magnético. La orientación de los edificios de $\mathrm{La}$ Escuera y El Chorrillo no se han incluido al desconocerse donde se encontraba la entrada. Como puede verse en la figura 2 a el patrón de orientación de todos los edificios de culto ibérico parece seguir una distribución aleatoria.

En la figura 2b, c y d intentamos comprobar si la imbricación de un santuario dentro del tejido urbano puede determinar su orientación. La figura $2 \mathrm{~b}$ muestra sólo aquellos edificios de culto insertos dentro de la trama urbana de un poblado, sin ocupar una situación singular aparente y clasificados como "domésticos gentilicios" o "dinásticos gentilicios" por Almagro Gorbea y Moneo (2000) situados principalmente en el área del Levante-Noreste peninsular, son los casos de: Alorda Park (santuario A), Burriac, Castellet de Bernabé (departamento 2), El Oral (edificio IIIJ), Moleta del Remei (recinto 7), Molí d'Espígol (estancia 65), Puntal dels Llops (estancias 1 y 14) y Sant Miquel de Llíria (estancia 14). Como vemos, este grupo de edificios muestra un patrón de orientación aparentemente aleatorio.

En la figura 2c mostramos la orientación de templos extraurbanos: Cancho Roano, Cerro de los Santos, Cigarralejo y La Luz. En este caso podemos comprobar que tres de los cuatro edificios se encuentran orientados dentro o cercanos a la zona del horizonte donde se producen los ortos del Sol y la Luna. La situación de la entrada del santuario de La Luz, el único que apunta al suroeste, no se conoce con seguridad pues no existe evidencia arquitectónica de ella. Nótese que si la entrada estuviese realmente en la pared oriental (girada $180^{\circ}$ en el diagrama) la orientación del edificio sería consistente con la del resto.

En la figura $2 \mathrm{~d}$ incluimos aquellos edificios cultuales que aun estando situados en núcleos de población se encuentran aislados y/o ocupan posiciones singulares dentro del poblado. Almagro Gorbea y Moneo (2000) clasifican estos edificios como "recintros sacros" (La Alcudia, Illeta des Banyets), de tipo "clásico" (Azaila A, Ullastret), de tipo "dinástico clientelar" (El Amarejo, La Alcudia), santuario "de entrada" (Torreparedones), o de tipo incierto (Bastida de les Alcuses). Como podemos ver en la figura, este grupo de edificios muestra un comportamiento similar al grupo de los santuarios extraurbanos. Existen varios casos en que no se sigue esta regla y que merecen comentarse. Dos datos de orientación corresponden a una de las entradas de santuarios que disponen de dos accesos $(\mathrm{La}$ Alcudia y Bastida de les Alcuses), siendo de destacar que la orientación de la otra entrada sí que es consistente con la tendencia mayoritaria de apuntar a levante. El resto corresponden al templo A de Illeta des Banyets, que podría tratarse realmente de un regia y el edificio de Torreparedones que se trata de un santuario de entrada adosado a otras construcciones.

En la figura 3 hemos introducido los santuarios extraurbanos y los urbanos no "gentilicios". Como podemos observar, la orientación mayoritaria es hacia donde se producen los ortos solares y lunares, diferente al patrón mostrado por los santuarios "gentilicios" (Fig. 2b). En nuestra opinión, los santuarios no "gentilicios" tenderían a estar orientados en una cierta dirección con significado astronómico, pero sólo y cuando no hubiese limitantes urbanos o de visibilidad, cosa que ocurre en los santuarios domésticos insertos en los poblados. Es posible que los elementos astronómicos sólo fuesen un elemento considerado por las élites religiosas y políticas de la sociedad, pero de escaso interés o importancia en las concepciones cultuales del pueblo en general. En un trabajo muy interesante, AlmagroGorbea (1996: 95) ya indica como característica de los templa, recintos sacros y santuarios "dinásticos" ibéricos su orientación astronómica, posiblemente heredera de tradiciones semitas. Este mismo autor sugiere que dicha orientación está "basada en concepciones cósmicas seguramente de tipo augural".

En la figura 4 incluimos patrones de orientación típicos de templos pertenecientes a otras culturas mediterráneas contemporáneas a la ibérica, como la romana, griega, etrusca y las norteafricanas prerromanas. Los datos sobre templos romanos provienen de edificios de culto de la Península Itálica (Aveni y Romano 1994) y de ciudades romanas del norte de África (Esteban et al. 2001). Los datos sobre templos griegos provienen de Aveni y Romano (1994), Papathanassiou y Hoskin (1994) y Aveni y Romano (2000). Las orientaciones de templos etruscos fueron obtenidas por Aveni y Romano 


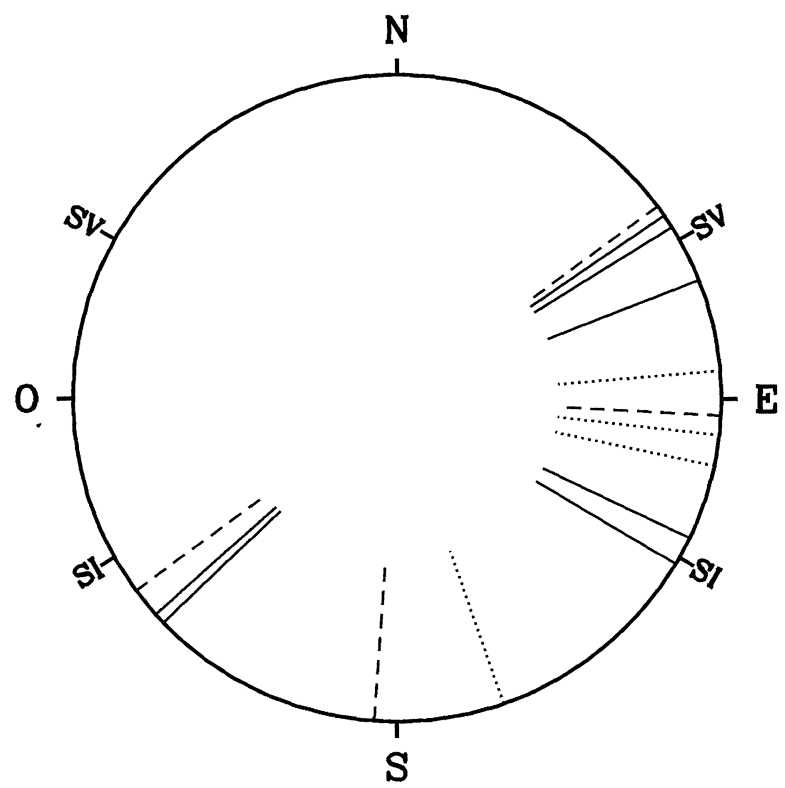

Fig. 3. Orientación de los edificios de culto ibérico tanto extraurbanos como urbanos de culto no "gentilicio". Significado de los tipos de línea: ver pie de figura 2.

(1994). La muestra de templos prerromanos norteafricanos proviene de Esteban y otros (2001) e incluye templos númidas, mauros, garamantes y el templo púnico de Kerkouane. Como vemos el patrón de orientación de los templos ibéricos es similar al griego y especialmente parecido al norteafricano prerromano, que está dominado por la orientación mayoritaria de los santuarios y templos dedicados a Baal Hamon y su interpretación romana como Saturno Africano. Los templos ibéricos, griegos y africanos comparten una orientación preferente dentro del intervalo de acimutes cubierto por los ortos del Sol y de la Luna. Por el contrario, los templos romanos son un claro ejemplo de conjunto con disposición aleatoria. Curiosamente, los edificios de culto etruscos apuntan de forma sistemática hacia la mitad meridional del horizonte.

Desgraciadamente, no disponemos de estudios sobre la orientación de una muestra significativa de edificios de culto fenicios y púnicos, aunque a través de referencias escritas de la antigüedad sabemos que los templos de Salomón en Jerusalén y de Melkart en Gades estaban orientados hacia levante. La arqueología también nos indica que ésta era la orientación de los templos de Astarté de Kitión (Karageorghis 1976: 118) y de Sarepta (Pritchard 1978). En el mundo púnico también parece común esta costumbre en la orientación de los edifi- cios sacros. Por ejemplo, el santuario neopúnico de Thinissut (Bir Bou Rekba, Túnez) se encuentra orientado fielmente a lo largo de los ejes cardinales (Merlin 1910). En Sicilia y Cerdeña podemos encontrar como los restos de los tofet y de las áreas sacrificiales y sacras púnicas se encuentran orientadas siguiendo los ejes cardinales (Ribichini y Xella 1994). Por otra parte, nuestras propias mediciones en el templo púnico urbano de Kerkouane en el Cabo Bon nos proporcionan el mismo resultado (Esteban et al. 2001).

\section{NECRÓPOLIS IBÉRICAS. EL CASO DE CABEZO LUCERO}

Las tumbas de las necrópolis ibéricas del sureste suelen disponerse a lo largo de unos ejes de simetría (García-Gelabert 1999). En Los Villares, Blánquez (1990: 123) encuentra que las tumbas tumulares se encuentran orientadas siguiendo los ejes cardinales; la misma disposición que se observa en Los Nietos (García Cano 1990). Uno de los casos más llamativos es el de la necrópolis de Cabezo Lucero donde la orientación general es este-oeste (Aranegui et al. 1984), disposición que también encontramos en la necrópolis cercana de La Albufereta (Rubio 1986: 382). Por otro lado, es frecuente que las falcatas se encuentren depositadas a lo largo de la direción este-oeste dentro de los enterramientos (Aranegui et al. 1982). Finalmente, esta regularidad en el trazado y ordenación de las necrópolis también se ha observado en yacimientos del noreste de Andalucía como en Estacar de Robarinas (García-Gelabert y Blázquez, 1992) y en Castellones de Ceal (Chapa y Pereira 1992).

La presencia de un patrón de orientación en las tumbas de las necrópolis, donde el eje este-oeste parece ser el dominante, y su similitud con el patrón mostrado por los edificios de culto discutida en la sección anterior sugiere que ambas características podrían obedecer a un mismo principio dentro de la cosmovisión ibérica, apoyando la tantas veces señalada relación íntima entre el mundo religioso y funerario en esta cultura. Por otra parte, según indican Almagro Gorbea y Moneo (2000: 138), algunos santuarios ibéricos, especialmente aquellos clasificados como "recintos sacros": caso de Illeta dels Banyets (B) y La Alcudia, están relacionados con elementos funerarios, características propias de los herôon, ambos edificios muestran orientación astronómica y una tipología semítica. 

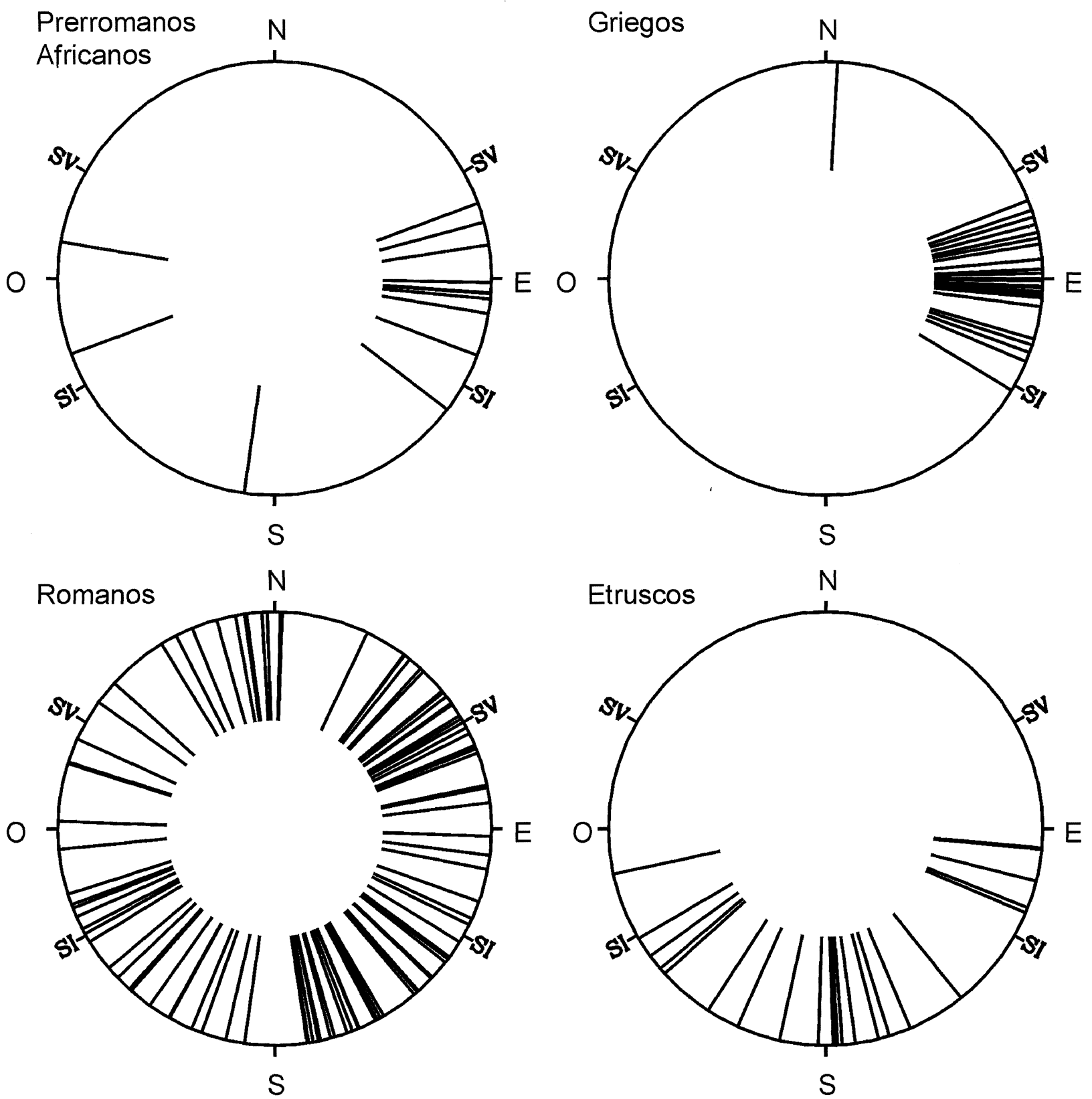

Fig. 4. Diagramas de orientación de templos de distintas culturas del Mediterráneo. Los datos han sido recopilados de la bibliografía (ver texto).

El patrón de orientación de las tumbas en las necrópolis ibéricas parece similar al que suelen mostrar las sepulturas fenicias del sur de la Península Ibérica del periodo u horizonte fenicio arcaico (siglos VIII a VII a.C.). En particular, las cámaras funerarias de Trayamar y Puente de Noy presentan sus accesos orientados hacia el este (Ramos Sainz 1986: 32-33). Belmonte (1999: fig. 5.5) encuentra que la mayoría de las tumbas de la necrópolis fenopúnica de Baria se encuentran orientadas hacia el orto solar del solsticio de invierno. Por otra parte y ya en territorio africano, nuestras propias medidas en la necrópolis arcaica de Útica (siglo VII a.C.) en Túnez, también son consistentes con este esquema (Belmonte et al. 1998). Sin embargo, más tarde en el periodo púnico (siglos VI a III a.C.) sólo 


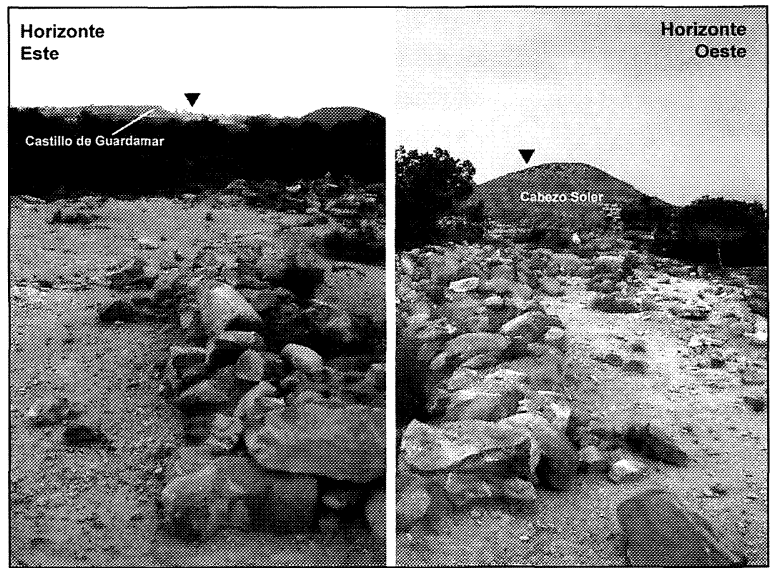

Lám. VII. Horizontes oriental y occidental visibles desde la zona central de la Necrópolis de Cabezo Lucero (tumbas G, $\mathrm{H}$ y M). Las flechas indican los puntos donde se produce el orto y ocaso solares en el punto medio temporal entre ambos solsticios (muy cercano a los equinoccios). En dicha fecha el orto se produce sobre la actual ciudad de Guardamar, cerca de la montaña donde se encontraba el santuario ibérico del Castillo de Guardamar. Hacia el oeste, el ocaso en dicho momento del año se produce sobre el Cabezo Soler, el elemento dominante del horizonte poniente. Resulta llamativo que las hiladas de piedras de los túmulos (visibles en ambas fotografías) se encuentran alineadas visualmente con este eje de clara significación astronómica, geográfica y posiblemente ritual.

la disposición de algunas tumbas de la Península Ibérica parece seguir este esquema. Según Ramos Sainz (1986: 32-33), este hecho podría indicar un cambio en el ritual funerario. Como vemos, si suponemos que la costumbre en la orientación de las tumbas de los cementerios ibéricos fue una herencia semita, ésta debió adquirirse en épocas tempranas, quizás en época orientalizante.

Cabezo Lucero (Guardamar del Segura, Alicante) es la única necrópolis ibérica estudiada dentro de nuestro proyecto hasta la fecha. El lugar ha sido excavado en varias ocasiones desde la década de los 80 (ver Aranegui 1992, y las referencias allí citadas). Se encuentra situado en una zona especialmente rica e interesante debido a la alta densidad de yacimientos ibéricos en sus cercanías y la presencia de un importante establecimiento fenicio a escasos kilómetros: La Fonteta-La Rábita, fechado al menos desde finales del siglo VIII a.C. (González Prats et al. 1997). El periodo de utilización de la necrópolis no fue muy extenso, abarcando desde el siglo $\mathrm{V}$ al IV a.C.

El horizonte visible desde la necrópolis es muy amplio, siendo el elemento más llamativo el cercano
Cabezo Soler, que se encuentra situado a una distancia de $1,5 \mathrm{~km}$ al oeste del yacimiento. Nuestras medidas nos indican que el ocaso del sol en los equinoccios se produce cerca de la cumbre de dicho cerro (ver Lám. VII). Por otra parte, su orto en esas mismas fechas se produce cerca de la montaña en la que se asienta el Castillo de Guardamar, donde se han encontrado evidencias de un santuario ibérico (Abad Casal 1992). Es especialmente llamativo que las tumbas se encuentren alineadas con el eje imaginario Cabezo Soler-Castillo de Guardamar, que corresponde además con el del orto-ocaso equinoccial. Como vemos, existe una posible relación entre hechos astronómicos, elementos del paisaje y orientación de tumbas, un entramado que, de repetirse en otros lugares, podría proporcionarnos nuevas claves para entender la organización del espacio ritual ibérico y quizás aspectos de su cosmovisión. En el futuro pretendemos ampliar el estudio arqueoastronómico a otras necrópolis ibéricas.

\section{DISCUSIÓN}

Nuestro estudio arqueoastronómico de 16 yacimientos ibéricos (15 santuarios y una necrópolis) ha proporcionado resultados que creemos muy significativos. En primer lugar, cuatro de ellos presentan marcadores o alineamientos precisos con el equinoccio (El Amarejo, La Serreta, La Carraposa, Cabezo Lucero) lo que supone un $25 \%$ del total. A este grupo se le podría añadir, bajo ciertas condiciones, hasta un máximo de cinco yacimientos más (La Alcudia, Cerro de los Santos, Bastida de les Alcuses, Coimbra del Barranco Ancho y El Chorrillo), con lo que alcanzaríamos un $56 \%$. En segundo lugar, tenemos tres santuarios cuya planta se encuentra alineada con los solsticios, en algún caso con una precisión muy notable (El Cigarralejo, templo B de Illeta des Banyets, Ullastret), a este grupo podría incluirse el posible edificio cultual de La Escuera. En tercer lugar, los alineamientos lunares son llamativos únicamente en el caso de El Cigarralejo y, quizás, en el conjunto 5 de La Bastida de les Alcuses, que comparten una misma orientación. Finalmente, tres de los yacimientos estudiados no parecen presentar relaciones astronómicas (La Luz, "templo" A de Illeta des Banyets y La Encarnación). La estadística total indica que siete de los 16 yacimientos estudiados, es decir un 44\%, presentan relaciones astronómicas relevantes, bien por la existencia de marcadores sobre elementos llamati- 
vos del horizonte o por la peculiar orientación de sus estructuras arquitectónicas. Este porcentaje podría aumentar hasta un máximo del $81 \%$ si incluimos el resto de yacimientos con relación astronómica "posible". Creemos que este resultado general indica que los elementos astronómicos fueron importantes (en algunos casos posiblemente determinantes) en el diseño y localización de una parte de los lugares de culto y quizás funerarios de los antiguos íberos.

Como todos sabemos, los equinoccios suponen el comienzo de la primavera y el otoño y, por lo tanto, el comienzo de las lluvias en el sureste español. La noción de equinoccio astronómico que usamos en la actualidad (Aparicio et al. 2000: 27; Belmonte 1999: 266) es un concepto abstracto que sólo tiene significado dentro de la astronomía geométrica que se desarrolló en Grecia entre los siglos IV y III a.C. En nuestra opinión, parece poco probable que este concepto fuese conocido por los antiguos íberos, incluso por sus élites culturales. Esta aparente paradoja tiene una sencilla solución si nos atenemos a conceptos sencillos y casi universales de ordenación del tiempo. Observacionalmente, los puntos de la trayectoria anual del Sol que pueden determinarse más fácilmente son los solsticios, que corresponden a los momentos en que el Sol tiene su orto u ocaso en los puntos más extremos norte y sur (Aparicio et al. 2000: 30). Uno de los conceptos de partición del año que parece haber sido utilizado en diferentes culturas prehistóricas del mundo es el del punto intermedio en tiempo entre ambos solsticios (de aquí en adelante usaremos la expresión "punto medio temporal" para abreviar); que permite una partición del año en cuatro periodos de igual duración (ver Ruggles 1997 para una discusión al respecto). Una cuestión importante es que el momento en que ocurre este "punto medio temporal" no coincide exactamente con el equinoccio astronómico sino que aquel se produce unas 45 horas antes del equinoccio de otoño y después del de primavera (la posición del Sol en dicho momento es de $\left.\delta=+0^{\circ} 44^{\prime}\right)$. Debido a que la posición del Sol es muy similar en el equinoccio astronómico y en el "punto medio temporal", es técnicamente muy difícil discriminar si un marcador o un alineamiento obedece a uno u otro evento, sobre todo si no tenemos una precisión del orden de varios minutos de arco o una muestra estadísticamente significativa de yacimientos.

¿Es posible que los yacimientos ibéricos en que hemos encontrado una relación con el equinoccio realmente quisieran indicar el "punto medio temporal"? Si recordamos, es justamente entre uno y dos días antes del equinoccio de otoño (ó después del de primavera) cuando los marcadores de El Amarejo, La Serreta y La Carraposa (quizás también el de Coimbra del Barranco Ancho) parecen ser más apropiados. En definitiva, tanto desde el punto de vista de su mayor simpleza conceptual como por las evidencias que nos proporcionan los marcadores astronómicos más precisos, creemos que el "punto medio temporal" debió ser probablemente el elemento astronómico buscado en los yacimientos "equinocciales" encontrados. Otro problema es dilucidar cuál equinoccio o "punto medio temporal" era el importante: ¿el de primavera o el de otoño? En este sentido, resulta útil el dato obtenido en el caso de El Amarejo, en el que parecería que el ritual se llevaría a cabo a comienzos de otoño debido al grado de madurez de las bellotas contenidas en las ofrendas.

Un hecho sugerente es el descubrimiento de un posible marcador del "punto medio temporal" en un templo dedicado originalmente a Baal Hamon en la ciudad de origen púnico-númida de Mactar, en la actual Túnez (Jiménez González et al. 1997; Esteban et al. 2001). Este templo muestra una orientación casi perfecta hacia el este geográfico y, además, el orto solar en el "punto medio temporal" se produce exactamente sobre una muesca visible sobre una montaña lejana. La hipótesis astronómica parece tener un refrendo en el hecho de que el templo fue reutilizado y dedicado al dios solar Apolo en época romana. Resulta llamativo que los marcadores astronómicos de los santuarios ibéricos de El Amarejo, La Serreta, La Carraposa y el del citado templo de Mactar son análogos. ¿Estamos ante un elemento ritual producto de la común influencia púnica a ambas orillas del Mediterráneo? Recordemos que el comienzo del calendario fenopúnico coincidía muy probablemente con el principio del otoño (Stieglitz 2000)

La existencia de los marcadores "equinocciales" encontrados, pudiera deberse a varios motivos. En primer lugar, su utilidad más directa pudo haber sido calendárica, como sistema práctico para la determinación de fiestas y para la ordenación del "tiempo social" de la colectividad. Distintas culturas de la antigüedad desarrollaron lo que denominamos "calendarios de horizonte" basados en el seguimiento, desde un lugar fijo generalmente con importancia religiosa, de la posición de los ortos u ocasos del Sol respecto a los distintos elementos to- 
pográficos del horizonte. Muy posiblemente, esta actividad sería una herramienta controlada directamente por las élites políticas y quizás esto explique que la orientación astronómica sea una característica de los santuarios dinásticos, templa y recintos sacros, directamente controlados por la élite, en contra de lo que observamos en los santuarios domésticos "gentilicios", donde la astronomía no parece ser un ingrediente importante. La utilización de "calendarios de horizonte" se ha evidenciado en multitud de culturas con distinto grado de desarrollo cultural y tecnológico, como la azteca y la maya (ver Aveni 1991) o las del suroeste de los EEUU (como la constatada etnográficamente entre los indios hopi, Forde 1931) y las megalíticas de las Islas Británicas. En segundo lugar, y de forma no necesariamente excluyente, el orto solar por un punto llamativo del horizonte bien pudo haber sido utilizado como elemento hierofánico en el ritual llevado a cabo en el santuario. Por otra parte, estos marcadores que utilizan elementos lejanos del horizonte permiten que el fenómeno sea visible en una área extensa alrededor del santuario, lo que sugiere quizás una dimensión pública al ritual asociado al fenómeno y a su fecha de ocurrencia, no dirigida a un grupo exclusivo, y quizá relacionado con romerías dirigidas al santuario en las fechas señaladas por el propio marcador.

\section{AGRADECIMIENTOS}

Es una gran suerte el haber contado con el apoyo y la colaboración de distintos profesionales de la arqueología como José Pérez Ballester, Emilio Cortell Pérez, Antonio Poveda Navarro y José Miguel García Cano, así como con los comentarios de Martín Almagro-Gorbea y Santiago Broncano. Tam-bién quisiera agradecer a Carmen Pinza y Santiago Esteban por su ayuda incondicional en tantas ocasiones y por hacer más agradable el trabajo de campo.

\section{BIBLIOGRAFÍA}

ABAD CASAL, L. 1992: "Terracotas ibéricas del Castillo de Guardamar". Estudios de arqueología ibérica y romana. Homenaje a Enrique Pla Ballester. Serie de Trabajos Varios SIP 89: 225-238.

ABAD CASAL, L. y SALA SELLÉS, F. 1997: "Sobre el posible uso cúltico de algunos edificios de la contesta- nia ibérica". Quaderns de Prehistòria i Arqueologia de Castelló 18: 91-102.

APARICIO, A.; ESTEBAN, C. y BELMONTE, J.A. 2000: "Las bases astronómicas: el cielo a simple vista". En J.A. Belmonte (coord.): Arqueoastronomía Hispana (segunda edición). Equipo Sirius S.A. Madrid: 19-65.

ALMAGRO-GORBEA, M. 1996: Ideología y poder en Tartessos y el mundo ibérico. Real Academia de la Historia. Madrid.

ALMAGRO-GORBEA, M. y DOMÍNGUEZ, A. 19881989: "El palacio de Cancho Roano y sus paralelos arquitectónicos y funcionales". Zephyrus 41-42: 339382.

ALMAGRO-GORBEA, M. y MONEO, T. 2000: Santuarios urbanos en el mundo ibérico. Real Academia de la Historia. Madrid.

ARANEGUI, C. 1992: "La necrópolis de Cabezo Lucero (Guardamar del Segura, Alicante)". Congreso de Arqueología Ibérica. Las Necrópolis. Serie Varia 1. Universidad Autónoma de Madrid: 169-188.

ARANEGUI, C.; JODIN, A.; LLOBREGAT, E.A.; ROUILLARD, P. y UROZ, J. 1982: "Fouilles du site ibérique de Cabezo Lucero (Guardamar del Segura, Alicante) Troisième campagne". Mélanges de la Casa de Velázquez XIX/1: 427-436.

- 1984: "Fouilles du site ibérique de Cabezo Lucero (Guardamar del Segura, Alicante) Quatrième campagne". Mélanges de la Casa de Velázquez XXI: 393-404.

AVENI, A.F. 1991: Observadores del cielo en el México antiguo. Fondo de Cultura Económica. México D.F.

AVENI, A.F. y ROMANO, G. 1994: "Orientation and Etruscan ritual”. Antiquity 68: 545-563.

- 2000: "Temple orientations in Magna Graecia and Sicily". Archaeoastronomy (Supplement to the Journal for the History of Astronomy), 25: 51-57.

BARBERÀ, J. y PASCUAL, R. 1979-1980: “Burriac, un yacimiento protohistórico de la costa catalana (Cabrera del Mar, Barcelona)". Ampurias 22-23: 183-219.

BELARTE, M. C. y SANMARTÍ, J. 1997: "Espais de culte i pràctiques rituals a la Catalunya protohistòrica". Quaderns de Prehistòria i Arqueologia de Castelló 18: 7-32.

BELMONTE, J.A. 1999: Las leyes del cielo. Ediciones Temas de Hoy, S.A. Madrid.

BELMONTE, J.A.; ESTEBAN, C. y JIMÉNEZ GONZÁLEZ, J.J. 1998: "Mediterranean archaeoastronomy and archaeotopography: pre-Roman tombs of Africa Proconsularis". Archaeoastronomy Supplement to the Journal for the History of Astronomy 23: 7-24.

BLÁNQUEZ PÉREZ, J. J. 1990: La formación del mundo ibérico en el sureste de la Meseta (estudio arqueológico de las necrópolis de la provincia de Albacete). Instituto de Estudios Albacentenses-CSIC. Albacete.

BONET, H. 1995: El Tossal de Sant Miquel de Llíria: la antigua Edeta y su territorio. Diputación de Valencia. Valencia.

T. P., 59, n. $^{\circ} 2,2002$ 
BONET, H.; MATA, C. y GUÉRIN, P. 1986: “Análisis microespacial del poblado ibérico de del Puntal dels Llops (Olocau, Valencia)". Arqueología Espacial, 9: 321-337.

BRONCANO, S. 1989: El depósito votivo ibérico de El Amarejo. Bonete (Albacete). Excavaciones Arqueológicas en España 156. Ministerio de Cultura. Madrid.

BRONCANO, S. y BLÁNQUEZ, J. 1985: El Amarejo (Bonete, Albacete). Excavaciones Arqueológicas en España 139. Ministerio de Cultura. Madrid.

CELESTINO PÉREZ, S.; JIMÉNEZ ÁVILA, J.; MARTÍN BAÑÓN, A.; HERNÁNDEZ CARRETERO, A. y PAVÓN SOLDEVILLA, I. 1996: El Palacio-Santuario de Cancho Roano V-VI-VII. Publicaciones del Museo Arqueológico Provincial de Badajoz. Madrid.

CHAPA, T. y PEREIRA, J. 1992: "La necrópolis de Castellones de Ceal (Hinojares, Jaén)". Congreso de Arqueología Ibérica. Las Necrópolis. Serie Varia 1. Universidad Autónoma de Madrid: 431-454.

CUADRADO DÍAZ, E. 1950: Excavaciones en el santuario ibérico de El Cigarralejo (Mula, Murcia). Informes y Memorias, 21. Ministerio de Educación Nacional. Madrid

DÍES CUSI, E. y ÁLVAREZ GARCÍA, N. 1997: “Análisis del conjunto 5 de La Bastida de les Alcuses (Mogente, Valencia): un edificio con posible funcionalidad cultual". Quaderns de Prehistòria i Arqueologia de Castelló 18:147-170.

ESTEBAN, C. 1999: “Astronomy and the ancient Iberians. Archaeoastronomical fieldwork in Iberian sacred enclosures". Actes de la V ${ }^{\text {ème }}$ Conférence de la SEAC (Danzig 1997). Swiatowit Supplement Series H: Anthropology II: 111-130.

- 2001: “Astronomía y religión ibérica". Revista de Arqueología 238: 12-19.

ESTEBAN, C.; BELMONTE, J.A.; PERERA BETANCORT, M.A.; MARRERO, R. y JIMÉNEZ GONZÁLEZ, J.J. 2001: "Orientations of pre-Islamic temples of the North-west of Africa", Archaeoastronomy (Supplement to the Journal for the History of Astronomy) 26: 65-84.

ESTEBAN, C. y CORTELL PÉREZ, E. 1997: “Consideraciones arqueoastronómicas sobre el santuario ibérico de La Serreta". Recerques del Museu d'Alcoi 6: 131-140.

ESTEBAN, C. y POVEDA NAVARRO, A. e.p.: "Análisis arqueoastronómico de algunos yacimientos del Valle del Vinalopó". Alebus.

FERNÁNDEZ CASTRO, M.C. y CUNLIFFE, B. 1988: Excavations at Torreparedones 1988. The Guadajoz Project. Institute of Archaeology. Oxford

FORDE, C.D. 1931: "Hopi Agriculture and Land Ownership". Journal of the Royal Anthropological Institute of Great Britain and Ireland 61: 357-405.

GARCÍA CANO, J.M. 1990: “Notas sobre la necrópolis ibérica de Los Nietos". Verdolay 2: 161-171.

GARCÍA CANO, J.M.; INIESTA SANMARTÍN, A. y
PAGE del POZO, V. 1991-1992: "El santuario ibérico de Coimbra del Barranco Ancho (Jumilla, Murcia)". Anales de Prehistoria y Arqueología 7-8: 75-82.

GARCÍA-GELABERT, M.P. 1999: "Rituales funerarios en el mundo ibérico". Religión y magia en la antigüedad (Valencia, 1997): 103-122. Valencia.

GARCÍA-GELABERT, M.P. y BLÁZQUEZ, J.M. 1992: "Las necrópolis oretanas de Cástulo. Paralelos con las necrópolis ibéricas del Sureste". Congreso de Arqueología Ibérica. Las Necrópolis. Serie Varia 1. Universidad Autónoma de Madrid: 455-472.

GONZÁLEZ PRATS, A.; GARCÍA MENARGUEZ, A. y RUÍZ SEGURA, E. 1997: "La Fonteta. Una ciudad fenicia en Occidente". Revista de Arqueología 190: 813.

GUSI, F. 1997: "Lugares sagrados, divinidades, cultos y rituales en el levante de Iberia". Quaderns de Prehistòria i Arqueologia de Castelló 18: 171:209.

JIMÉNEZ GONZÁLEZ, J.J.; ESTEBAN. C. y BELMONTE, J.A. 1997: “Arqueoastronomía en el África Proconsular". Revista de Arqueología 203: 46-53.

KARAGEORGHIS, V. 1976: View from the Bronze Age. Mycenaean and Phoenician Discoveries at Kition. E.P. Dutton \& Co., Inc. New York.

KRUPP, E.C. 1989: “Un firmamento para cada estación”. En E.C. Krupp (coord.): En busca de las antiguas astronomías. Ediciones Pirámide. Madrid: 25-57.

LILLO CARPIO, P. A. 1993-1994. "Notas sobre el templo del santuario de la Luz (Murcia)". Anales de Prehistoria y Arqueología 9-10: 155-174.

LLOBREGAT CONESA, E.A. 1985: El conjunto de templos ibéricos de la Illeta dels Banyets (El Campello). Studia Historica in Honorem Vicente Morellá. Diputación Provincial. Alicante.

LLOBREGAT CONESA, E.A.; CORTELL PÉREZ, E.; JUAN MOLTÓ, J. y SEGURA MARTÍ, J.M. 1992: “El Urbanismo ibérico en La Serreta”. Recerques del Museu d'Alcoi 1: 37-70.

MALUQUER, J. 1986: Molí d'Espígol. Tornabous. Guies de jaciments arqueològics. Barcelona.

MÁRQUEZ VILLORA, J.C.; POVEDA NAVARRO, A. M.; SOLER GARCÍA, M.D. y TORRES SALINAS, F. J. 1999: "El edificio ibérico del yacimiento de El Chorrillo (Elda-Petrer-Sax, Alicante)". Actas del XXIV Congreso Nacional de Arqueología (Cartagena 1997): 327-336.

MERLIN, A. 1910: Le sanctuaire de Baal et de Tanit près de Siagu. Paris.

MIRÓ I ALAIX, Mª T. 1990: "Les màscares del temple d'Ullastret". Zephyrus XLIII: 305-309.

MORET, P. 1996: Les fortifications ibériques: de la fin de l'âge de Bronze à la conquête romaine. Collection de la Casa de Velázquez 56. Madrid.

NORDSTRÖM, S. 1967: Excavaciones en el poblado ibérico de La Escuera (San Fulgencio, Alicante). Serie de Trabajos Varios del SIP, 34. Valencia. 
OLCINA DOMÉNECH, M.; GRAU MIRA, F.; SALA SELLÉS, F.; MOLTÓ GISBERT, S.; REIG SEGUÍ, C. y SEGURA MARTÍ, J.M. 1998: "Nuevas aportaciones a la evolución de la ciudad ibérica: el ejemplo de La Serreta". Saguntum, Extra-1: 47-54.

PAPATHANASSIOU, M. y HOSKIN, M. 1994: "Orientations of the Greek temples on Corfu". Journal for the History of Astronomy 25: 111-114.

PÉREZ BALLESTER, J. y BORREDÁ MEJÍAS, R. e.p.: "La Carraposa (Rotglà i Corbera - Llanera de Ranes). Un lugar de culto ibérico en el Valle del Canyoles (La Costera, Valencia)". Madrider Mitteilungen.

PRITCHARD, J.B. 1978: Recovering Sarepta, a Phoenician City. Princeton.

RAMALLO ASENSIO, S. F. 1991: "Un santuario de época tardorepublicana en la Encarnación, Caravaca, Murcia". Cuadernos de Arqueología Romana 1: 36-64.

RAMOS FERNÁNDEZ, R. 1995: El Templo Ibérico de La Alcudia. La Dama de Elche. Ajuntament d'Elx.

RAMOS SAINZ, M. L. 1986: Estudio sobre el ritual funerario en las necrópolis fenicias y púnicas de la Península Ibérica. Ediciones de la Universidad Autónoma de Madrid. Madrid.
RIBICHINI, S. y XELLA, P. 1994: La religione fenicia e punica in Italia. Libreria dello Stato. Istituto Poligrafico e Zecca dello Stato. Roma.

RUANO RUÍZ, E. y SAN NICOLÁS DEL TORO, M. 1990: "Exvotos ibéricos procedentes de La Encarnación (Caravaca, Murcia)". Verdolay 2: 101-107.

RUBIO, F. 1986: La necrópolis ibérica de la Albufereta de Alicante (Valencia, España). Academia de Cultura Valenciana, Serie arqueológica 11. Valencia.

RUGGLES, C.L.N. 1997: "Whose equinox?”. Archaeoastronomy (Supplement to the Journal for the History of Astronomy) 22: 45-49.

SAVIRÓN, P. 1875: "Noticias de varias excavaciones del Cerro de los Santos". Revista de Archivos, Bibliotecas y Museos V:125-129, 161-164, 193-197, 229-234, 245247.

SCHAEFER, B.E. 2001: "New Methods and Techniques for Historical Astronomy and Archaeoastronomy", Archaeoastronomy. The Journal for Astronomy in Culture 15: 121-136.

STIEGLITZ, R R. 2000: "The Phoenician-Punic Calendar". Actas del IV Congreso Internacional de Estudios Fenicios y Púnicos, II: 691-695. Cádiz. 